ANNALES

POLONICI MATHEMATICI

$92.2(2007)$

\title{
Difference methods for parabolic functional differential problems of the Neumann type
}

by K. Kropielnicka (Gdańsk)

\begin{abstract}
Nonlinear parabolic functional differential equations with initial boundary conditions of the Neumann type are considered. A general class of difference methods for the problem is constructed. Theorems on the convergence of difference schemes and error estimates of approximate solutions are presented. The proof of the stability of the difference functional problem is based on a comparison technique. Nonlinear estimates of the Perron type with respect to the functional variable for given functions are used. Numerical examples are given.
\end{abstract}

1. Introduction. For any two metric spaces $X$ and $Y$ we denote by $C(X, Y)$ the class of all continuous functions defined on $X$ and taking values in $Y$. Let $M[n]$ denote the set of all $n \times n$ real matrices. We will use vectorial inequalities, understanding that the same inequalities hold between the corresponding components. Let $E=[0, a] \times[-b, b]$, where $a>0$, $b=\left(b_{1}, \ldots, b_{n}\right), b_{i}>0$ for $1 \leq i \leq n$, and

$$
\partial_{0} E=[0, a] \times([-b, b] \backslash(-b, b)) .
$$

Write $\Sigma=E \times C(E, \mathbb{R}) \times \mathbb{R}^{n} \times M[n]$ and

$$
\partial_{0} E_{j}=\left\{(t, x) \in \partial_{0} E: x_{j}=b_{j}\right\} \cup\left\{(t, x) \in \partial_{0} E: x_{j}=-b_{j}\right\}, \quad 1 \leq j \leq n,
$$

and suppose that

$$
f: \Sigma \rightarrow \mathbb{R}, \quad \varphi:[-b, b] \rightarrow \mathbb{R}, \quad \varphi_{j}: \partial_{0} E_{j} \rightarrow \mathbb{R}, \quad 1 \leq j \leq n,
$$

are given functions. We consider the functional differential equation

$$
\partial_{t} z(t, x)=f\left(t, x, z, \partial_{x} z(t, x), \partial_{x x} z(t, x)\right)
$$

together with the initial boundary condition of Neumann type

2000 Mathematics Subject Classification: 65M12, 35R10.

Key words and phrases: functional differential equations, difference methods, stability and convergence. 


$$
\begin{aligned}
z(0, x) & =\varphi(x) & & \text { for } x \in[-b, b], \\
\partial_{x_{j}} z(t, x) & =\varphi_{j}(t, x) & & \text { for }(t, x) \in \partial_{0} E_{j}, 1 \leq j \leq n,
\end{aligned}
$$

where $\partial_{x} z=\left(\partial_{x_{1}} z, \ldots, \partial_{x_{n}} z\right)$, and $\partial_{x x} z=\left[\partial_{x_{i} x_{j}} z\right]_{i, j=1, \ldots, n}$.

For $t \in[0, a]$ we write $E_{t}=[0, t] \times[-b, b]$. The function $f$ is said to satisfy the Volterra condition if for each $(t, x, q, s) \in E \times \mathbb{R}^{n} \times M[n]$ and $z, \bar{z} \in$ $C(E, \mathbb{R})$ such that $z(\tau, y)=\bar{z}(\tau, y)$ for $(\tau, y) \in E_{t}$ we have $f(t, x, z, q, s)=$ $f(t, x, \bar{z}, q, s)$. Note that the Volterra condition means that the value of $f$ at the point $(t, x, z, q, s)$ of the space $\Sigma$ depends on $(t, x, q, s)$ and on the restriction of $z$ to the set $E_{t}$.

In this paper we assume that $f$ satisfies the Volterra condition and we consider classical solutions of (1)-(3).

We are interested in approximating classical solutions to problem (1)-(3) by solutions of associated difference functional equations and in estimation of the difference between these solutions.

Finite difference approximations of initial boundary value problems for parabolic differential or functional equations were considered by many authors under various assumptions. Difference methods for nonlinear parabolic differential equations with initial boundary conditions of the Dirichlet type were considered in [4], [6], [8]. Numerical treatment of the Cauchy problem can be found in [1], [5], [10], [16].

The paper [3] is concerned with initial boundary value problems of the Neumann type.

Difference methods for nonlinear parabolic equations with nonlinear boundary conditions are investigated in [7], [9], [14], [15].

The papers [11]-[13] deal with numerical methods for the reaction-diffusion-convection equations. The implicit difference methods proposed in those papers have the following property: almost linear parabolic equations without mixed derivatives are transformed into nonlinear systems of difference equations which require some kind of iterative schemes for the computation of numerical solutions. Various monotone iterative methods are presented in [11]-[13].

The main problem in the study of explicit difference methods is to find a difference or difference functional equation which is stable and satisfies consistency conditions with respect to the original problem.

The method of difference inequalities or theorems on linear recurrent inequalities are used in the study of stability of difference schemes. The proofs of convergence are also based on error estimates of approximate solutions to functional difference equations of the Volterra type with initial boundary conditions and with an unknown function of several variables.

It is usually assumed that the given functions have partial derivatives with respect to all variables except for $(t, x)$. Our assumptions are more 
general. We introduce nonlinear estimates of the Perron type with respect to the functional variable. Note that our theorems are new also in the case of parabolic equations without a functional variable.

The paper is organized as follows. In Section 2 we construct a class of difference schemes for (1)-(3). Error estimates for approximate solutions of difference functional problems are proved in Section 3. In Section 4 we prove a theorem on the convergence of a difference method for (1)-(3). This is the main part of the paper. Numerical examples are presented in the last section.

Differential equations with deviated variables and differential integral problems can be obtained from our general model by a natural specification of operators.

2. Discretization of mixed problems. We will denote by $F(X, Y)$ the class of all functions defined on $X$ and taking values in $Y$, where $X$ and $Y$ are arbitrary sets. For $x, y \in \mathbb{R}^{n}$ where $x=\left(x_{1}, \ldots, x_{n}\right), y=\left(y_{1}, \ldots, y_{n}\right)$, we write $\|x\|=\left|x_{1}\right|+\cdots+\left|x_{n}\right|$ and $x * y=\left(x_{1} y_{1}, \ldots, x_{n} y_{n}\right)$.

We now formulate a difference problem corresponding to (1)-(3). We define a mesh on $E$ in the following way. Let $\left(h_{0}, h^{\prime}\right)$ where $h^{\prime}=\left(h_{1}, \ldots, h_{n}\right)$ stand for steps of the mesh. For $h=\left(h_{0}, h^{\prime}\right)$ and $(r, m) \in \mathbb{Z}^{1+n}$ where $m=\left(m_{1}, \ldots, m_{n}\right)$ we define nodal points as follows:

$$
t^{(r)}=r h_{0}, \quad x^{(m)}=m * h^{\prime}, \quad x^{(m)}=\left(x_{1}^{\left(m_{1}\right)}, \ldots, x_{n}^{\left(m_{n}\right)}\right) .
$$

Let $H$ be the set of all $h=\left(h_{0}, h^{\prime}\right)$ such that there exists $\left(N_{1}, \ldots, N_{n}\right)=$ $N \in \mathbb{N}^{n}$ satisfying the condition $N * h^{\prime}=b$. We write $\|h\|=h_{0}+h_{1}+\cdots+h_{n}$. Let $N_{0} \in \mathbb{N}$ be defined by the relation $N_{0} h_{0} \leq a \leq\left(N_{0}+1\right) h_{0}$. For $h \in H$ we put

$$
\mathbb{R}_{h}^{1+n}=\left\{\left(t^{(r)}, x^{(m)}\right):(r, m) \in \mathbb{Z}^{1+n}\right\}
$$

and

$$
\begin{aligned}
E_{h} & =E \cap \mathbb{R}_{h}^{1+n}, \quad \partial_{0} E_{h}=\partial_{0} E \cap \mathbb{R}_{h}^{1+n}, \\
\partial_{0} E_{h . j} & =\partial_{0} E_{j} \cap \mathbb{R}_{h}^{1+n}, \quad j=1, \ldots, n, \\
E_{h}^{\prime} & =\left\{\left(t^{(r)}, x^{(m)}\right) \in E_{h}: 0 \leq r \leq N_{0}-1\right\}, \\
\Sigma_{h} & =E_{h}^{\prime} \times F\left(E_{h}, \mathbb{R}\right) \times \mathbb{R}^{n} \times M[n] .
\end{aligned}
$$

For $z: E_{h} \rightarrow \mathbb{R}$ we write $z^{(r, m)}=z\left(t^{(r)}, x^{(m)}\right)$. Put $E_{h . r}=E_{h} \cap\left(\left[0, t^{(r)}\right] \times \mathbb{R}^{n}\right)$, where $0 \leq r \leq N_{0}$, and

$$
\|z\|_{h . r}=\max \left\{\left|z^{(\widetilde{r}, m)}\right|:\left(t^{(\widetilde{r})}, x^{(m)}\right) \in E_{h . r}\right\}, \quad 0 \leq r \leq N_{0} .
$$

Let $e_{i}=(0, \ldots, 0,1,0, \ldots, 0) \in \mathbb{R}^{n}$ be the vector with 1 in the $i$ th position. Write

$$
J=\{(i, j): 1 \leq i, j \leq n, i \neq j\}
$$


and suppose that we have defined sets $J_{+}, J_{-} \subset J$ such that $J_{+} \cup J_{-}=J$, $J_{+} \cap J_{-}=\emptyset$ (in particular, it may happen that $J_{+}=\emptyset$ or $J_{-}=\emptyset$ ). We assume that $(i, j) \in J_{+}$when $(j, i) \in J_{+}$.

For each $m \in \mathbb{Z}^{n}$ such that $x^{(m)} \in[-b, b] \backslash(-b, b)$ we consider the class of $\alpha=\left(\alpha_{1}, \ldots, \alpha_{n}\right) \in \mathbb{Z}^{n}$ satisfying the conditions:

(i) $\|\alpha\|=1$ or $\|\alpha\|=2$,

(ii) if $m=\left(m_{1}, \ldots, m_{n}\right)$ and there is $j, 1 \leq j \leq n$, such that $m_{j}=N_{j}$ then $\alpha_{j} \in\{0,1\}$,

(iii) if $m=\left(m_{1}, \ldots, m_{n}\right)$ and there is $j, 1 \leq j \leq n$, such that $m_{j}=-N_{j}$ then $\alpha_{j} \in\{-1,0\}$.

The set of $\alpha \in \mathbb{Z}^{n}$ satisfying the above conditions will be denoted by $A^{(m)}$. Define

$$
\begin{aligned}
\partial E_{h}^{+} & =\left\{\left(t^{(r)}, x^{(m+\alpha)}\right): 0 \leq i \leq N_{0},\left(t^{(r)}, x^{(m)}\right) \in \partial_{0} E_{h} \text { and } \alpha \in A^{(m)}\right\}, \\
E_{h}^{+} & =\partial E_{h}^{+} \cup E_{h} .
\end{aligned}
$$

Let $z: E_{h}^{+} \rightarrow \mathbb{R}$ and $-N \leq m \leq N$. We define

$$
\delta_{i}^{+} z^{(r, m)}=\frac{1}{h_{i}}\left(z^{\left(r, m+e_{i}\right)}-z^{(r, m)}\right), \quad \delta_{i}^{-} z^{(r, m)}=\frac{1}{h_{i}}\left(z^{(r, m)}-z^{\left(r, m-e_{i}\right)}\right),
$$

where $1 \leq i \leq n$. We apply the difference operators $\delta_{0}$ and $\delta=\left(\delta_{1}, \ldots, \delta_{n}\right)$ given by

$$
\begin{aligned}
\delta_{0} z^{(r, m)} & =\frac{1}{h_{0}}\left(z^{(r+1, m)}-z^{(r, m)}\right), \\
\delta_{i} z^{(r, m)} & =\frac{1}{2}\left(\delta_{i}^{+} z^{(r, m)}+\delta_{i}^{-} z^{(r, m)}\right), \quad 1 \leq i \leq n .
\end{aligned}
$$

The difference operator $\delta^{(2)}=\left[\delta_{i j}\right]_{i, j=1, \ldots, n}$ of the second order is defined in the following way:

$$
\delta_{i i} z^{(r, m)}=\delta_{i}^{+} \delta_{i}^{-} z^{(r, m)} \quad \text { for } 1 \leq i \leq n
$$

and

$$
\begin{array}{ll}
\delta_{i j} z^{(r, m)}=\frac{1}{2}\left(\delta_{i}^{+} \delta_{j}^{-} z^{(r, m)}+\delta_{i}^{-} \delta_{j}^{+} z^{(r, m)}\right) & \text { for }(i, j) \in J_{-}, \\
\delta_{i j} z^{(r, m)}=\frac{1}{2}\left(\delta_{i}^{+} \delta_{j}^{+} z^{(r, m)}+\delta_{i}^{-} \delta_{j}^{-} z^{(r, m)}\right) & \text { for }(i, j) \in J_{+} .
\end{array}
$$

Suppose that the functions

$$
f_{h}: \Sigma_{h} \rightarrow \mathbb{R}, \quad \varphi_{h}:[-b, b] \rightarrow \mathbb{R}, \quad \varphi_{h . j}: \partial_{0} E_{h . j} \rightarrow \mathbb{R}, \quad 1 \leq j \leq n,
$$


are given. We consider the difference equations

$$
\begin{aligned}
& \delta_{0} z^{(r, m)}=f_{h}\left(t^{(r)}, x^{(m)}, z, \delta z^{(r, m)}, \delta^{(2)} z^{(r, m)}\right), \quad-N \leq m \leq N, \\
& z\left(t^{(r)}, x^{(m+\alpha)}\right)=z\left(t^{(r)}, x^{(m-\alpha)}\right) \\
& +2 \sum_{j=1}^{n} \alpha_{j} h_{j} \varphi_{h . j}\left(t^{(r)}, x^{(m)}\right) \quad \text { on } \partial_{0} E_{h}, \alpha \in A^{(m)},
\end{aligned}
$$

with the initial condition

$$
z^{(0, m)}=\varphi_{h}^{(m)} \quad \text { for } x^{(m)} \in[-b, b] .
$$

We assume that $f_{h}$ satisfies the Volterra condition: for each $\left(t^{(r)}, x^{(m)}, q, s\right)$ $\in \Sigma_{h}^{\prime} \times \mathbb{R}^{n} \times M[n]$ and $z, \bar{z} \in F\left(E_{h}, \mathbb{R}\right)$ such that $z(\tau, y)=\bar{z}(\tau, y)$ for $(\tau, y) \in E_{h . r}$ we have

$$
f_{h}\left(t^{(r)}, x^{(m)}, z, q, s\right)=f_{h}\left(t^{(r)}, x^{(m)}, \bar{z}, q, s\right) .
$$

Then there exists exactly one solution $u_{h}: E_{h}^{+} \rightarrow \mathbb{R}$ of problem (4)-(6).

3. Approximate solutions of difference functional problems. We will denote by $F_{h}$ the Nemytskiı operator corresponding to (4), i.e.

$$
F_{h}[z]^{(r, m)}=f_{h}\left(t^{(r)}, x^{(m)}, z, \delta z^{(r, m)}, \delta^{(2)} z^{(r, m)}\right), \quad\left(t^{(r)}, x^{(m)}\right) \in E_{h}^{\prime} .
$$

Suppose that $u_{h}: E_{h}^{+} \rightarrow \mathbb{R}$ is the solution of problem (4)-(6) and $v_{h}: E_{h}^{+} \rightarrow \mathbb{R}$ satisfies the following conditions:

$$
\begin{gathered}
\left|\delta_{0} v_{h}^{(r, m)}-F_{h}\left[v_{h}\right]^{(r, m)}\right| \leq \gamma(h) \quad \text { on } E_{h}^{\prime}, \\
\left|v_{h}^{(r, m+\alpha)}-v_{h}^{(r, m-\alpha)}-2 \sum_{j=1}^{n} \alpha_{j} h_{j} \varphi_{j . h}^{(r, m)}\right| \leq \gamma_{1}(h)\left\|h^{\prime}\right\|^{2} \quad \text { on } \partial_{0} E_{h}, \alpha \in A^{(m)}, \\
\left|\varphi^{(m)}-\varphi_{h}^{(m)}\right| \leq \gamma_{0}(h), \quad x^{(m)} \in[-b, b],
\end{gathered}
$$

where $\gamma, \gamma_{0}, \gamma_{1}: H \rightarrow \mathbb{R}_{+}$and

$$
\lim _{h \rightarrow 0} \gamma(h)=0, \quad \lim _{h \rightarrow 0} \gamma_{0}(h)=0, \quad \lim _{h \rightarrow 0} \gamma_{1}(h)=0 .
$$

The function $v_{h}$ satisfying the above relations is considered to be an approximate solution of problem (4)-(6). We prove an estimate of the difference between the exact and approximate solutions of (4)-(6). Put

$$
\left.I_{h}=\left\{t^{(r)}: 0 \leq r \leq N_{0}\right)\right\}, \quad I_{h}^{\prime}=I_{h} \backslash\left\{t^{\left(N_{0}\right)}\right\}
$$

For a function $\eta: I_{h} \rightarrow \mathbb{R}$ we write $\eta^{(r)}=\eta\left(t^{(r)}\right)$.

Assumption $\mathrm{H}\left[\sigma_{h}\right]$. The function $\sigma_{h}: I_{h}^{\prime} \times \mathbb{R}_{+} \rightarrow \mathbb{R}_{+}$is such that

1) $\sigma_{h}$ is nondecreasing with respect to the second variable and $\sigma_{h}(t, 0)$ $=0$ for $t \in I_{h}^{\prime}$, 
2) the difference problem

$$
\begin{aligned}
\eta^{(r+1)} & =\eta^{(r)}+h_{0} \sigma_{h}\left(t^{(r)}, \eta^{(r)}\right), \quad 0 \leq r \leq N_{0}-1, \\
\eta^{(0)} & =0,
\end{aligned}
$$

is stable in the following sense: if $\bar{\gamma}, \bar{\gamma}_{0}: H \rightarrow \mathbb{R}_{+}$are functions such that

$$
\lim _{h \rightarrow 0} \bar{\gamma}(h)=0, \quad \lim _{h \rightarrow 0} \bar{\gamma}_{0}(h)=0
$$

and $\eta_{h}: I_{h} \rightarrow \mathbb{R}_{+}$is a solution of the difference problem

$$
\begin{aligned}
\eta^{(r+1)} & =\eta^{(r)}+h_{0} \sigma_{h}\left(t^{(r)}, \eta^{(r)}\right)+h_{0} \bar{\gamma}(h), \quad 0 \leq r \leq N_{0}-1, \\
\eta^{(0)} & =\bar{\gamma}_{0}(h),
\end{aligned}
$$

then there is $\widetilde{\alpha}: H \rightarrow \mathbb{R}_{+}$such that $\eta_{h}^{(r)} \leq \widetilde{\alpha}(h)$ for $t^{(r)} \in I_{h}$ and $\lim _{h \rightarrow 0} \widetilde{\alpha}(h)=0$.

Assumption $\mathrm{H}\left[f_{h}\right]$. The function $f_{h}: \Sigma_{h} \rightarrow \mathbb{R}$ of variables $(t, x, w, q, s)$, where $q=\left(q_{1}, \ldots, q_{n}\right), s=\left[s_{i j}\right]_{i, j=1, \ldots, n}$, satisfies the conditions:

1) $f_{h}(t, x, z, \cdot, \cdot) \in C\left(\mathbb{R}^{n} \times M[n], \mathbb{R}\right)$ and the derivatives

$$
\partial_{q} f_{h}=\left(\partial_{q_{1}} f_{h}, \ldots, \partial_{q_{n}} f_{h}\right), \quad \partial_{s} f_{h}=\left[\partial_{s_{i j}} f_{h}\right]_{i, j=1, \ldots, n},
$$

exist on $\Sigma_{h}$ and $\partial_{q} f_{h}(t, x, z, \cdot, \cdot) \in C\left(\mathbb{R}^{n} \times M[n], \mathbb{R}^{n}\right), \partial_{s} f_{h}(t, x, z, \cdot, \cdot)$ $\in C\left(\mathbb{R}^{n} \times M[n], M[n]\right)$ for each $(t, x, z) \in E_{h}^{\prime} \times F\left(E_{h}, \mathbb{R}\right)$,

2) the matrix $\partial_{s} f_{h}$ is symmetric and

$$
\begin{aligned}
& \partial_{s_{i j}} f_{h}(P) \geq 0 \text { for }(i, j) \in J_{+}, \partial_{s_{i j}} f_{h}(P) \leq 0 \quad \text { for }(i, j) \in J_{-}, \\
& 1-2 h_{0} \sum_{j=1}^{n} \frac{1}{h_{j}^{2}} \partial_{s_{j j}} f_{h}(P)+h_{0} \sum_{(i, j) \in J} \frac{1}{h_{i} h_{j}}\left|\partial_{s_{i j}} f_{h}(P)\right| \geq 0, \\
& -\frac{1}{2}\left|\partial_{q_{i}} f_{h}(P)\right|+\frac{1}{h_{i}} \partial_{s_{i i}} f_{h}(P)-\sum_{j=1, j \neq i} \frac{1}{h_{j}}\left|\partial_{s_{i j}} f_{h}(P)\right| \geq 0, \quad 1 \leq i \leq n,
\end{aligned}
$$

where $P=(x, y, z, q, s) \in \Sigma_{h}$,

3) there is a function $\sigma_{h}$ satisfying Assumption $\mathrm{H}\left[\sigma_{h}\right]$ such that

$$
\left\|f_{h}(t, x, z, q, s)-f_{h}(t, x, \bar{z}, q, s)\right\| \leq \sigma_{h}\left(t,\|z-\bar{z}\|_{h . r}\right)
$$

on $\Sigma_{h}$.

REMARK 1. It is assumed in condition 2) of $\mathrm{H}\left[f_{h}\right]$ that the functions

$$
g_{h . i j}=\operatorname{sign} \partial_{s_{i j}} f_{h}, \quad(i, j) \in J,
$$

are constant on $\Sigma_{h}$. Relations (15) can be considered as definitions of the sets $J_{+}$and $J_{-}$. 
REMARK 2. Suppose that

(i) conditions 1), 2) of Assumption $\mathrm{H}\left[f_{h}\right]$ are satisfied,

(ii) there is $\widetilde{p}>0$ such that

$$
\partial_{s_{i i}} f_{h}(P)-\sum_{\substack{j=1 \\ j \neq i}}^{n}\left|\partial_{s_{i j}} f_{h}(P)\right| \geq \widetilde{p}, \quad i=1, \ldots, n,
$$

where $P=(t, x, z, q, s) \in \Sigma_{h}$.

Then there is $\widetilde{\varepsilon}>0$ such that for $\left\|h^{\prime}\right\|<\widetilde{\varepsilon}$ assumption (17) is satisfied.

TheOREm 3.1. Suppose that Assumption $\mathrm{H}\left[f_{h}\right]$ is satisfied and

1) $u_{h}: E_{h}^{+} \rightarrow \mathbb{R}$ is a solution of (4)-(6) and the function $v_{h}: E_{h}^{+} \rightarrow \mathbb{R}$ satisfies (7)-(9),

2) there is $\widetilde{c}>0$ such that $\left\|h^{\prime}\right\|^{2} \leq \widetilde{c} h_{0}$.

Then there is $\alpha: H \rightarrow \mathbb{R}_{+}$such that

$$
\left|\left(u_{h}-v_{h}\right)^{(r, m)}\right| \leq \alpha(h) \quad \text { on } E_{h}
$$

and

$$
\lim _{h \rightarrow 0} \alpha(h)=0 .
$$

Proof. Let $\Gamma_{h}: E_{h}^{\prime} \rightarrow \mathbb{R}, \Gamma_{0 . h}: E_{0 . h} \rightarrow \mathbb{R}$, and $\Gamma_{\partial . h}: \partial_{0} E_{h} \rightarrow \mathbb{R}$ be defined by the relations

$$
\begin{gathered}
\delta_{0} v_{h}^{(r, m)}=F_{h}\left[v_{h}\right]^{(r, m)}+\Gamma_{h}^{(r, m)} \quad \text { on } E_{h}^{\prime}, \\
v_{h}^{(r, m+\alpha)}-v_{h}^{(r, m-\alpha)}=2 \sum_{j=1}^{n} \alpha_{j} h_{j} \varphi_{j . h}^{(r, m)}+\Gamma_{\partial . h}^{(r, m)} \quad \text { on } \partial_{0} E_{h}, \quad \alpha \in A^{(m)}, \\
v_{h}^{(0, m)}=\varphi_{h}^{(m)}+\Gamma_{0 . h}^{(m)}, \quad x^{(m)} \in[-b, b] .
\end{gathered}
$$

It follows from (7)-(10) that

$$
\begin{gathered}
\left|\Gamma_{h}^{(r, m)}\right| \leq \gamma(h) \quad \text { on } E_{h}^{\prime}, \quad\left|\Gamma_{\partial . h}^{(r, m)}\right| \leq \gamma_{1}(h)\left\|h^{\prime}\right\|^{2} \quad \text { on } \partial_{0} E_{h}, \\
\left|\Gamma_{0 . h}^{(m)}\right| \leq \gamma_{0}(h) \quad \text { for } x^{(m)} \in[-b, b]
\end{gathered}
$$

and

$$
\lim _{h \rightarrow 0} \gamma(h)=0, \quad \lim _{h \rightarrow 0} \gamma_{0}(h)=0, \quad \lim _{h \rightarrow 0} \gamma_{1}(h)=0 .
$$

Write $z_{h}=u_{h}-v_{h}$ and

$$
\begin{aligned}
\Xi_{h}^{(r, m)}= & z_{h}^{(r, m)}+h_{0}\left[f_{h}\left(t^{(r)}, x^{(m)}, v_{h}, \delta u_{h}^{(r, m)}, \delta^{(2)} u_{h}^{(r, m)}\right)\right. \\
& \left.-f_{h}\left(t^{(r)}, x^{(m)}, v_{h}, \delta v_{h}^{(r, m)}, \delta^{(2)} v_{h}^{(r, m)}\right)\right], \\
\Lambda_{h}^{(r, m)}= & \left(t^{(r)}, x^{(m)}, u_{h}, \delta u_{h}^{(r, m)}, \delta^{(2)} u_{h}^{(r, m)}\right) \\
& -f_{h}\left(t^{(r)}, x^{(m)}, v_{h}, \delta u_{h}^{(r, m)}, \delta^{(2)} u_{h}^{(r, m)}\right) .
\end{aligned}
$$


Thus we have

$$
z_{h}^{(r+1, m)}=\Xi_{h}^{(r, m)}+h_{0} \Lambda_{h}^{(r, m)}-h_{0} \Gamma_{h}^{(r, m)} \quad \text { on } E_{h}^{\prime}
$$

and

$$
z_{h}^{(r, m+\alpha)}=z_{h}^{(r, m-\alpha)}+\Gamma_{\partial . h}^{(r, m)} \quad \text { on } \partial_{0} E_{h}, \quad \alpha \in A^{(m)} .
$$

We first estimate $\Xi_{h}^{(r, m)}$. Write

$$
\begin{aligned}
A_{i .+}(Q) & =\frac{1}{2 h_{i}} \partial_{q_{i}} f_{h}(Q)+\frac{1}{h_{i}^{2}} \partial_{s_{i i}} f_{h}(Q)-\sum_{\substack{j=1 \\
j \neq i}}^{n} \frac{1}{h_{i} h_{j}}\left|\partial_{s_{i j}} f_{h}(Q)\right|, \\
A_{i .-}(Q) & =-\frac{1}{2 h_{i}} \partial_{q_{i}} f_{h}(Q)+\frac{1}{h_{i}^{2}} \partial_{s_{i i}} f_{h}(Q)-\sum_{\substack{j=1 \\
j \neq i}}^{n} \frac{1}{h_{i} h_{j}}\left|\partial_{s_{i j}} f_{h}(Q)\right|, \\
B(Q) & =1-2 \sum_{i=1}^{n} \frac{h_{0}}{h_{i}^{2}} \partial_{s_{i i}} f_{h}(Q)+\sum_{(i, j) \in J}^{n} \frac{h_{0}}{h_{i} h_{j}}\left|\partial_{s_{i j}} f_{h}(Q)\right|, \\
C_{i}(Q) & =\sum_{\substack{j=1 \\
j \neq i}}^{n} \frac{1}{h_{i} h_{j}}\left|\partial_{s_{i j}} f_{h}(Q)\right|,
\end{aligned}
$$

where $Q \in \Sigma_{h}$ and $1 \leq i \leq n$. It follows from the definition of difference operators and from condition 1) of Assumption $H\left[f_{h}\right]$ that there is $Q \in \Sigma_{h}$ such that

$$
\begin{aligned}
& \Xi_{h}^{(r, m)}= B(Q) z_{h}^{(r, m)} \\
&+h_{0} \sum_{i=1}^{n} A_{i .+}(Q) z_{h}^{\left(r, m+e_{i}\right)}+h_{0} \sum_{i=1}^{n} A_{i .-}(Q) z_{h}^{\left(r, m-e_{i}\right)} \\
&+h_{0} \sum_{i=1}^{n} C_{i}(Q)\left[z_{h}^{\left(r, m+e_{i}+e_{j}\right)}+z_{h}^{\left(r, m-e_{i}-e_{j}\right)}+z_{h}^{\left(r, m+e_{i}-e_{j}\right)}+z_{h}^{\left(r, m-e_{i}+e_{j}\right)}\right]
\end{aligned}
$$

where $\left(t^{(r)}, x^{(m)}\right) \in E_{h}^{\prime}$. We conclude from (15)-(17) that

$$
A_{i .+}(Q) \geq 0, \quad A_{i .-}(Q) \geq 0, \quad B(Q) \geq 0, \quad C_{i}(Q) \geq 0 .
$$

It is important in our considerations that

$$
h_{0} \sum_{i=1}^{n} A_{i .+}(Q)+h_{0} \sum_{i=1}^{n} A_{i .-}(Q)+B^{(r, m)}+2 h_{0} \sum_{i=1}^{n} C_{i}(Q)=1 .
$$

Write

$$
\begin{aligned}
& \left.\varepsilon_{h}^{(r)}=\max \left\{\left|z_{h}^{(r, m)}\right|:\left(t^{(r)}, x^{(m)}\right) \in E_{h . r}\right)\right\}, \\
& \widetilde{\varepsilon}_{h}^{(r)}=\max \left\{\left|z_{h}^{(r, m)}\right|:\left(t^{(r)}, x^{(m)}\right) \in E_{h}^{+} \cap\left(\left[0, t^{(r)}\right] \times \mathbb{R}^{n}\right)\right\},
\end{aligned}
$$


where $0 \leq r \leq N_{0}$. It follows from (19)-(20) that

$$
\left|\Xi_{h}^{(r, m)}\right| \leq \widetilde{\varepsilon}_{h}^{(r)} \quad \text { on } E_{h}^{\prime} .
$$

Next, according to condition 3) of Assumption $\mathrm{H}\left[f_{h}\right]$ we have

$$
\left|\Lambda_{h}^{(r, m)}\right| \leq \sigma_{h}\left(t^{(r)},\|z\|_{h . r}\right) \quad \text { on } E_{h}^{\prime} .
$$

The above estimates and (18) imply

$$
\varepsilon_{h}^{(r+1)} \leq \widetilde{\varepsilon}_{h}^{(r)}+h_{0} \sigma_{h}\left(t^{(r)}, \varepsilon_{h}^{(r)}\right)+h_{0} \gamma(h),
$$

where $0 \leq r \leq N_{0}-1$. It is easily seen that

$$
\widetilde{\varepsilon}_{h}^{(r)} \leq \varepsilon_{h}^{(r)}+h_{0} \gamma_{1}(h) \widetilde{c}, \quad 0 \leq r \leq N_{0}-1 .
$$

Thus we see that the function $\varepsilon_{h}$ satisfies the recurrent inequality

$$
\varepsilon_{h}^{(r+1)} \leq \varepsilon_{h}^{(r)}+h_{0} \sigma_{h}\left(t^{(r)}, \varepsilon_{h}^{(r)}\right)+h_{0}\left(\gamma(h)+\widetilde{c} \gamma_{1}(h)\right), \quad 0 \leq r \leq N_{0}-1,
$$

and $\varepsilon_{h}^{(0)} \leq \gamma_{0}(h)$. Let us denote by $\bar{\eta}_{h}: I_{h} \rightarrow \mathbb{R}_{+}$the solution of

$$
\begin{aligned}
\eta_{h}^{(r+1)} & =\eta_{h}^{(r)}+h_{0} \sigma_{h}\left(t^{(r)}, \eta_{h}^{(r)}\right)+h_{0}\left(\gamma(h)+\widetilde{c} \gamma_{1}(h)\right), \quad 0 \leq r \leq N_{0}-1, \\
\eta_{h}^{(0)} & =\gamma_{0}(h) .
\end{aligned}
$$

It follows easily that $\varepsilon_{h}^{(r)} \leq \eta_{h}^{(r)}$ for $0 \leq r \leq N_{0}$. Then the assertion of the theorem follows from the stability of problem (11), (12).

4. Convergence of implicit difference methods. For any $z \in C(E, \mathbb{R})$ we put

$$
\|z\|_{t}=\max \left\{|z(\tau, x)|:(\tau, x) \in E_{t}\right\}, \quad 0 \leq t \leq a .
$$

Now we give an example of an operator $f_{h}$ associated with (1)-(3), and we prove that the corresponding difference method is convergent.

Equation (1) contains the function variable $z$ which is an element of the space $C(E, \mathbb{R})$. So we need an interpolating operator $T_{h}: F\left(E_{h}, \mathbb{R}\right) \rightarrow$ $C(E, \mathbb{R})$. We give an example of such an operator as follows. Put

$$
\Im=\left\{\lambda=\left(\lambda_{1}, \ldots, \lambda_{n}\right): \lambda_{i} \in\{0,1\} \text { for } 0 \leq i \leq n\right\} .
$$

Let $z \in F\left(E_{h}, \mathbb{R}\right)$ and $(t, x) \in E, t \leq N_{0} h_{0}$. There exists $(r, m) \in \mathbb{Z}^{1+n}$ such that $t^{(r)} \leq t \leq t^{(r+1)}, x^{(m)} \leq x \leq x^{(m+1)}$ and $\left(t^{(r)}, x^{(m)}\right),\left(t^{(r+1)}, x^{(m+1)}\right) \in$ $E_{h}$ where $m+1=\left(m_{1}+1, \ldots, m_{n}+1\right)$. We define

$$
\begin{aligned}
T_{h}[z](t, x)= & \frac{t-t^{(r)}}{h_{0}} \sum_{\lambda \in \Im} z^{(r+1, m+\lambda)}\left(\frac{x-x^{(m)}}{h^{\prime}}\right)^{\lambda}\left(1-\frac{x-x^{(m)}}{h^{\prime}}\right)^{1-\lambda} \\
& +\left(1-\frac{t-t^{(r)}}{h_{0}}\right) \sum_{\lambda \in \Im} z^{(r, m+\lambda)}\left(\frac{x-x^{(m)}}{h^{\prime}}\right)^{\lambda}\left(1-\frac{x-x^{(m)}}{h^{\prime}}\right)^{1-\lambda}
\end{aligned}
$$


where

$$
\begin{aligned}
\left(\frac{x-x^{(m)}}{h^{\prime}}\right)^{\lambda} & =\prod_{i=1}^{n}\left(\frac{x_{i}-x_{i}^{\left(m_{i}\right)}}{h_{i}}\right)^{\lambda_{i}}, \\
\left(1-\frac{x-x^{(m)}}{h^{\prime}}\right)^{1-\lambda} & =\prod_{i=1}^{n}\left(1-\frac{x_{i}-x_{i}^{\left(m_{i}\right)}}{h_{i}}\right)^{1-\lambda_{i}},
\end{aligned}
$$

and we take $0^{0}=1$ in the above formulas. If $(t, x) \in E$ and $N_{0} h_{0}<t \leq a$ then we put $T_{h}[z](t, x)=T_{h}[z]\left(N_{0} h_{0}, x\right)$. Thus we have defined $T_{h} z$ on $E$. It follows easily that $T_{h} z \in C(E, \mathbb{R})$, and that $\left\|T_{h}[z]\right\|_{t^{(r)}}=\|z\|_{h . r}, 0 \leq r \leq N_{0}$.

The above operator $T_{h}$ was first applied in [2] to construct difference methods for first order partial differential functional equations.

We approximate solutions of (1)-(3) with solutions of the difference equation

$$
\delta_{0} z^{(r, m)}=f\left(t^{(r)}, x^{(m)}, T_{h}[z], \delta z^{(r, m)}, \delta^{(2)} z^{(r, m)}\right)
$$

with initial boundary condition (5), (6).

Lemma 1. Suppose that $z: E \rightarrow \mathbb{R}$ and

1) $z(t, \cdot):[-b, b] \rightarrow \mathbb{R}$ is of class $C^{2}$ for $t \in[0, a]$ and $z_{h}=\left.z\right|_{E_{h}}$,

2) $\widetilde{d} \in \mathbb{R}_{+}$is a constant such that

$$
\left|\partial_{x_{j} x_{k}} z(t, x)\right| \leq \widetilde{d}, \quad(t, x) \in E, \quad j, k=1, \ldots, n,
$$

3) there is $L \in \mathbb{R}_{+}$such that

$$
|z(t, x)-z(\bar{t}, x)| \leq L|t-\bar{t}| .
$$

Then

$$
\left\|T_{h}\left[z_{h}\right]-z\right\|_{t} \leq L h_{0}+\widetilde{d}\left\|h^{\prime}\right\|^{2}, \quad t \in\left[0, N_{0} h_{0}\right] .
$$

Proof. Let $(t, x) \in E$ and $t^{(r)} \leq t \leq t^{(r+1)}, x^{(m)} \leq x \leq x^{(m+1)}$ where $\left(t^{(r)}, x^{(m)}\right),\left(t^{(r+1)}, x^{(m+1)}\right) \in E_{h}$. Write

$$
\begin{aligned}
U(t, x)= & \frac{t-t^{(r)}}{h_{0}} \\
& \times\left\{\sum_{\lambda \in \Im} z^{(r+1, m+\lambda)}\left(\frac{x-x^{(m)}}{h^{\prime}}\right)^{\lambda}\left(1-\frac{x-x^{(m)}}{h^{\prime}}\right)^{1-\lambda}-z\left(t^{(r+1)}, x\right)\right\}, \\
V(t, x)= & \left(1-\frac{t-t^{(r)}}{h_{0}}\right) \\
& \times\left\{\sum_{\lambda \in \Im} z^{(r, m+\lambda)}\left(\frac{x-x^{(m)}}{h^{\prime}}\right)^{\lambda}\left(1-\frac{x-x^{(m)}}{h^{\prime}}\right)^{1-\lambda}-z\left(t^{(r)}, x\right)\right\} \\
W(t, x)= & \frac{t-t^{(r)}}{h_{0}}\left[z\left(t^{(r+1)}, x\right)-z(t, x)\right]+\left(1-\frac{t-t^{(r)}}{h_{0}}\right)\left[z\left(t^{(r)}, x\right)-z(t, x)\right] .
\end{aligned}
$$


Thus we have

$$
T_{h}[z](t, x)-z(t, x)=U(t, x)+V(t, x)+W(t, x) .
$$

It follows from $([2$, Theorem 5.27]) that

$$
|U(t, x)|+|V(t, x)| \leq \widetilde{d}\left\|h^{\prime}\right\|^{2} .
$$

According to condition (22) we have $|W(t, x)| \leq L h_{0}$. Hence, the proof is complete.

Assumption $\mathrm{H}[\sigma]$. The function $\sigma:[0, a] \times \mathbb{R}_{+} \rightarrow \mathbb{R}_{+}$is such that

1) if $(t, p),(\bar{t}, \bar{p}) \in[0, a] \times \mathbb{R}_{+}$and $t \leq \bar{t}, p \leq \bar{p}$ then $\sigma(t, p) \leq \sigma(\bar{t}, \bar{p})$,

2) $\sigma(t, 0)=0$ for $t \in[0, a]$, and the maximal solution of the Cauchy problem

$$
\zeta^{\prime}(t)=\sigma(t, \zeta(t)), \quad \zeta(0)=0,
$$

is $\zeta(t)=0$ for $t \in[0, a]$.

Assumption $\mathrm{H}[f]$. The function $f: \Sigma \rightarrow \mathbb{R}$ of variables $(t, x, z, q, s)$ satisfies the conditions:

1) $f(t, x, z, \cdot, \cdot) \in C\left(\mathbb{R}^{n} \times M[n], \mathbb{R}\right)$, the derivatives

$$
\partial_{q} f=\left(\partial_{q_{1}} f, \ldots, \partial_{q_{n}} f\right), \quad \partial_{s} f=\left[\partial_{s_{i j}} f\right]_{i, j=1, \ldots, n},
$$

exist on $\Sigma$ and

$$
\begin{aligned}
& \partial_{q} f(t, x, z, \cdot, \cdot) \in C\left(\mathbb{R}^{n} \times M[n], \mathbb{R}^{n}\right), \\
& \partial_{s} f(t, x, z, \cdot, \cdot) \in C\left(\mathbb{R}^{n} \times M[n], M[n]\right)
\end{aligned}
$$

for each $(t, x, z) \in E^{\prime} \times F\left(E_{h}, \mathbb{R}\right)$,

2) the matrix $\partial_{s} f$ is symmetric and

$$
\begin{aligned}
& \partial_{s_{i j}} f(P) \geq 0 \quad \text { for }(i, j) \in J_{+}, \quad \partial_{s_{i j}} f(P) \leq 0 \quad \text { for }(i, j) \in J_{-} \\
& 1-2 h_{0} \sum_{j=1}^{n} \frac{1}{h_{j}^{2}} \partial_{s_{j j}} f(P)+h_{0} \sum_{(i, j) \in J} \frac{1}{h_{i} h_{j}}\left|\partial_{s_{i j}} f(P)\right| \geq 0, \\
& -\frac{1}{2}\left|\partial_{q_{i}} f(P)\right|+\frac{1}{h_{i}} \partial_{s_{i i}} f(P)-\sum_{j=1, j \neq i} \frac{1}{h_{j}}\left|\partial_{s_{i j}} f(P)\right| \geq 0, \quad 1 \leq i \leq n,
\end{aligned}
$$

where $P=(t, x, z, q, s) \in \Sigma$,

3 ) there is a function $\sigma$ satisfying Assumption $\mathrm{H}[\sigma]$ such that

$$
\|f(t, x, z, q, s)-f(t, x, \bar{z}, q, s)\| \leq \sigma\left(t,\|z-\bar{z}\|_{t}\right)
$$

on $\Sigma_{h}$.

We can now formulate our main results.

THEOREM 4.1. Suppose that Assumption $\mathrm{H}[f]$ is satisfied and $\Omega \subset \mathbb{R}^{1+n}$ is an open and bounded set such that $E \subset \Omega$ and

1) the function $v: \Omega \rightarrow \mathbb{R}$ is the solution of (1)-(3), 
2) $v(\cdot, x)$ is of class $C^{1}$ and $v(t, \cdot)$ is of class $C^{2}$,

3) the function $u_{h}: E_{h}^{+} \rightarrow \mathbb{R}$ is a solution of (5), (6), (21), where $\varphi_{h . j}=\left.\varphi_{j}\right|_{\partial_{0} E_{h}}$ for $1 \leq j \leq n$ and there is $\gamma_{0}: H \rightarrow \mathbb{R}_{+}$such that

$$
\left|\varphi_{0}^{(m)}-\varphi_{h}^{(m)}\right| \leq \gamma_{0}(h) \quad \text { for } x^{(m)} \in[-b, b] \quad \text { and } \quad \lim _{h \rightarrow 0} \gamma_{0}(h)=0,
$$

4) there exist $c, \widetilde{c}>0$ such that $h_{k} \leq h_{j}$ for $1 \leq k, j \leq n$, and $\left\|h^{\prime}\right\|^{2} \leq$ $\widetilde{c} h_{0}$.

Then there exist $\varepsilon_{0}>0$ and a function $\alpha: H \rightarrow \mathbb{R}_{+}$such that for $\|h\|<\varepsilon_{0}$, $h \in H$ we have

$$
\left|\left(u_{h}-v_{h}\right)^{(r, m)}\right| \leq \alpha(h) \quad \text { on } E_{h} \quad \text { and } \quad \lim _{h \rightarrow 0} \alpha(h)=0,
$$

where $v_{h}$ is the restriction of $v$ to $E_{h}$.

Proof. We will use Theorem 3.1 on the error estimation. Write $f_{h}(t, x, z, q, s)=f\left(t, x, T_{h}[z], q, s\right) \quad$ on $\Sigma_{h}, \quad \sigma_{h}(t, p)=\sigma(t, p) \quad$ on $I_{h}^{\prime} \times \mathbb{R}_{+}$. It follows that conditions (7)-(9) are satisfied. Now we prove that problem (11), (12) is stable. Let $\eta_{h}: I_{h} \rightarrow \mathbb{R}_{+}$be a solution of (13), (14) where $\bar{\gamma}_{0}, \bar{\gamma}: H \rightarrow \mathbb{R}_{+}$and $\lim _{h \rightarrow 0} \bar{\gamma}_{0}(h)=\lim _{h \rightarrow 0} \bar{\gamma}(h)=0$. Let $\widetilde{\eta}_{h}:[0, a] \rightarrow \mathbb{R}_{+}$ be the maximal solution of the Cauchy problem

$$
\zeta^{\prime}(t)=\sigma(t, \zeta(t))+\bar{\gamma}(h), \quad \zeta(0)=\alpha_{0}(h) .
$$

Then $\lim _{h \rightarrow 0} \widetilde{\eta}_{h}(t)=0$ uniformly on $[0, a]$. The function $\widetilde{\eta}_{h}$ is convex on $[0, a]$, therefore we have

$$
\widetilde{\eta}_{h}^{(r+1)} \geq \widetilde{\eta}_{h}^{(r)}+h_{0} \sigma\left(t^{(r)}, \widetilde{\eta}_{h}^{(r)}\right)+h_{0} \bar{\gamma}(h), \quad 0 \leq r \leq N_{0}-1 .
$$

Since $\eta_{h}$ satisfies (13) we have $\eta_{h}^{(r)} \leq \widetilde{\eta}_{h}^{(r)} \leq \widetilde{\eta}_{h}(a)$ for $0 \leq i \leq N_{0}$, which completes the proof of the stability of problem (11), (12). It follows from Assumption $\mathrm{H}[f]$ that

$$
\begin{aligned}
\mid f_{h}(t, x, z, q, s) & -f_{h}(t, x, \bar{z}, q, s)|=| f\left(t, x, T_{h}[z], q, s\right)-f\left(t, x, T_{h}[\bar{z}], q, s\right) \mid \\
\leq & \sigma\left(t,\left\|T_{h}[z]-T_{h}[\bar{z}]\right\|_{t}\right) \leq \sigma\left(t,\|z-\bar{z}\|_{h . r}\right)=\sigma_{h}\left(t,\|z-\bar{z}\|_{h . r}\right) .
\end{aligned}
$$

Thus all the assumptions of Theorem 3.1 are satisfied and the proof of (23) is complete.

Remark 3. Suppose that Assumption $\mathrm{H}[f]$ is satisfied with

$$
\sigma(t, p)=L p, \quad(t, p) \in[0, a] \times \mathbb{R}_{+}, \quad \text { where } L \in \mathbb{R}_{+}
$$

and $f$ satisfies the Lipschitz condition with respect to the function variable. Then we obtain the following error estimates:

$$
\left\|u_{h}^{(i, m)}-v_{h}^{(i, m)}\right\| \leq \alpha_{0}(h) e^{L a}+\bar{\gamma}(h) \frac{e^{L a}-1}{L} \quad \text { on } E_{h} \text { if } L>0
$$


and

$$
\left\|u_{h}^{(i, m)}-v_{h}^{(i, m)}\right\| \leq \alpha_{0}(h)+a \bar{\gamma}(h) \quad \text { on } E_{h} \text { if } L=0 .
$$

The above inequality follows from $(23)$ with $\alpha(h)=\widetilde{\eta}_{h}(a)$ where $\widetilde{\eta}_{h}$ : $[0, a] \rightarrow \mathbb{R}_{+}$is a solution of $(24)$.

\section{Numerical examples}

EXAMPLE 1. Write

$$
\begin{aligned}
E & =[0,0.2] \times[-1,1] \times[-1,1], \\
\partial_{0} E & =[0,0.2] \times[([-1,1] \times[-1,1]) \backslash((-1,1) \times(-1,1))] .
\end{aligned}
$$

Consider the differential equation with deviated variables

$$
\begin{aligned}
\partial_{t} z(t, x, y)= & \partial_{x x} z(t, x, y)+\partial_{y y} z(t, x, y)+\frac{1}{4} \partial_{x y} z(t, x, y) \\
& +z(t, x, y)+z\left(t, \frac{x+y}{2}, \frac{x-y}{2}\right)+f(t, x, y)
\end{aligned}
$$

and the initial boundary conditions

$$
\begin{aligned}
z(0, x, y)=0 & \text { for }(x, y) \in[-1,1] \times[-1,1], \\
\partial_{x} z(t, x, y)=0 & \text { for } t \in[0,0.2], y \in[-1,1], x=1 \text { or } x=-1, \\
\partial_{y} z(t, x, y)=0 & \text { for } t \in[0,0.2], x \in[-1,1], y=1 \text { or } y=-1,
\end{aligned}
$$

where

$$
\begin{aligned}
& f(t, x, y) \\
& =4\left(1-e^{t}\right)\left(\left(3 x^{2}-1\right)\left(y^{2}-1\right)^{2}+\left(3 y^{2}-1\right)\left(x^{2}-1\right)^{2}+x y\left(x^{2}-1\right)\left(y^{2}-1\right)\right) \\
& \quad+\left(x^{2}-1\right)^{2}\left(y^{2}-1\right)^{2}+\left(1-e^{t}\right)\left(\left(x^{2}-y^{2}\right)^{2}-8\left(x^{2}+y^{2}-2\right)\right)^{2}: 16^{2}
\end{aligned}
$$

The function

$$
v(t, x, y)=\left(1-e^{t}\right)\left(x^{2}-1\right)^{2}\left(y^{2}-1\right)^{2}
$$

is a solution of problem (25)-(28).

We found approximate solutions of (25)-(28) using our numerical method, and taking the following steps of the mesh: $h_{0}=0.00005, h_{1}=0.02$, $h_{2}=0.02$.

Let $u_{h}$ denote the approximate solution of $(25)-(28)$ which is obtained by the difference scheme.

The average errors of the method are

$$
\varepsilon_{h}^{(r)}=\frac{1}{\left(2 N_{1}+1\right)\left(2 N_{2}+1\right)} \sum_{i=-N_{1}}^{N_{1}} \sum_{j=-N_{2}}^{N_{2}}\left|u_{h}^{(r, i, j)}-v_{h}^{(r, i, j)}\right|
$$

where $N_{1} h_{1}=1, N_{2} h_{2}=1$ and $v_{h}$ is the restriction of $v$ to the mesh. 
A list of the average errors $\varepsilon_{h}$ for fixed $t^{(r)}$ is given in Table 1.

Table 1. Errors $\varepsilon_{h}$

\begin{tabular}{|c|c|}
\hline$t^{(r)}$ & $\varepsilon_{h}^{(r)}$ \\
\hline$t=0.01$ & 0.00002 \\
\hline$t=0.05$ & 0.00028 \\
\hline$t=0.10$ & 0.00105 \\
\hline$t=0.15$ & 0.00222 \\
\hline$t=0.20$ & 0.00375 \\
\hline
\end{tabular}

The differential equation (25) contains the deviated variables $\left(t, \frac{x+y}{2}, \frac{x-y}{2}\right)$ and the example has the following property: if $\left(t^{(r)}, x^{\left(m_{1}\right)}, y^{\left(m_{2}\right)}\right)$ is a grid point then

$$
\left(t^{(r)}, 0.5\left(x^{\left(m_{1}\right)}+y^{\left(m_{2}\right)}\right), 0.5\left(x^{\left(m_{1}\right)}-y^{\left(m_{2}\right)}\right)\right)
$$

is not in general a grid point. We approximate the value $z\left(t^{(r)}, 0.5\left(x^{\left(m_{1}\right)}\right.\right.$ $\left.\left.+y^{\left(m_{2}\right)}\right), 0.5\left(x^{\left(m_{1}\right)}-y^{\left(m_{2}\right)}\right)\right)$ using the interpolating operator $T_{h}$ with $n=2$.

EXAMPLE 2. Consider the integral differential equation

$$
\begin{aligned}
& \partial_{t} z(t, x, y)=\partial_{x x} z(t, x, y)+\partial_{y y} z(t, x, y)+\partial_{x y} z(t, x, y) \\
& +\frac{\pi^{4}}{4} \int_{-x}^{x} \int_{-y}^{y} z(t, \tau, s) d s d \tau+\int_{0}^{t} z(\tau, x, y) d \tau+2 \pi^{2} z(t, x, y)+\cos \pi x \cos \pi y
\end{aligned}
$$

with the initial boundary conditions

$$
\begin{aligned}
z(0, x, y)=0 & \text { for }(x, y) \in[-1,1] \times[-1,1], \\
\partial_{x} z(t, x, y)=0 & \text { for } t \in[0,0.2], y \in[-1,1], x=1 \text { or } x=-1, \\
\partial_{y} z(t, x, y)=0 & \text { for } t \in[0,0.2], x \in[-1,1], y=1 \text { or } y=-1,
\end{aligned}
$$

The function $v(t, x, y)=\sin t \cos \pi x \cos \pi y$ is a solution of (30)-(33).

Let $u_{h}$ denote the approximate solution of (30)-(33) which is obtained by the difference scheme. The average errors of the method $\varepsilon_{h}^{(r)}$ are defined by (29), where $v_{h}$ is the restriction of $v$ to the mesh.

We found approximate solutions of (30)-(33) using the numerical method, and taking the following steps of the mesh: $h_{0}=0.00005, h_{1}=$ $0.02, h_{2}=0.02$. The method is also convergent and gives the average errors as in Table 2 . 
Table 2. Errors $\varepsilon_{h}$

$\left(h_{0}=0.00005, h_{1}=0.02, h_{2}=0.02\right)$

\begin{tabular}{cc}
\hline$t^{(r)}$ & $\varepsilon_{h}^{(r)}$ \\
\hline$t=0.01$ & 0.0000004 \\
$t=0.05$ & 0.0000098 \\
$t=0.10$ & 0.0000396 \\
$t=0.15$ & 0.0000972 \\
$t=0.20$ & 0.0001695 \\
\hline
\end{tabular}

The differential equation (30) contains integrals of the unknown function $z$. The corresponding difference equation includes the terms

$$
\int_{-x^{\left(m_{1}\right)}}^{x^{\left(m_{1}\right)}} \int_{-y^{\left(m_{2}\right)}}^{y^{\left(m_{2}\right)}} z\left(t^{(r)}, \tau, s\right) d s d \tau \text { and } \int_{0}^{t^{(r)}} z\left(\tau, x^{\left(m_{1}\right)}, y^{\left(m_{2}\right)}\right) d \tau
$$

where $z\left(t^{(r)}, x^{\left(m_{1}\right)}, y^{\left(m_{2}\right)}\right)$ is a grid point. The above integrals are approximated by

$$
\int_{-x^{\left(m_{1}\right)}}^{x_{-y}^{\left(m_{1}\right)}} \int_{y^{\left(m_{2}\right)}}^{y^{\left(m_{2}\right)}} T_{h}\left[z_{h}\right]\left(t^{(r)}, \tau, s\right) d s d \tau \quad \text { and } \quad \int_{0}^{t^{(r)}} T_{h}\left[z_{h}\right]\left(\tau, x^{\left(m_{1}\right)}, y^{\left(m_{2}\right)}\right) d \tau
$$

where $z_{h}$ is a solution of the difference equation. The above method is equivalent to the trapezoidal rule.

The computation was performed on a PC computer. Numerical results are consistent with our mathematical theory.

In difference schemes obtained by discretization of problem (1)-(3) a large number of previous values $z^{(r, m)}$ must be preserved, because they are needed to compute an approximate solution with $t=t^{(r+1)}$.

\section{References}

[1] P. Besala, Finite difference approximation to the Cauchy problem for nonlinear parabolic differential equations, Ann. Polon. Math. 46 (1985), 19-26.

[2] Z. Kamont, Hyperbolic Functional Differential Inequalities and Applications, Kluwer, Dordrecht, 1999.

[3] Z. Kamont and M. Kwapisz, Difference methods for nonlinear parabolic differentialfunctional systems with initial boundary conditions of the Neumann type, Comment. Math. Prace Mat. 28 (1989), 223-248.

[4] Z. Kamont and H. Leszczyński, Stability of difference equations generated by parabolic differential-functional problems, Rend. Mat. Appl. (7) 16 (1996), 265-287.

[5] H. Leszczyński, General finite difference approximation to the Cauchy problem for nonlinear parabolic differential-functional equations, Ann. Polon. Math. 53 (1991), $15-28$. 
[6] H. Leszczyński, Convergence of one-step difference methods for nonlinear parabolic differential-functional systems with initial boundary conditions of Dirichlet type, Comment. Math. Prace Mat. 30 (1991), 357-375.

[7] M. Malec, Schéma explicite des différences finies pour un système d'équations non linéaires du type parabolique avec des conditions aux limites non linéaires, Ann. Polon. Math. 41 (1983), 185-192.

[8] —, Sur une méthode des différences finies pour une équation non linéaire différentielle fonctionnelle aux dérivées mixtes, ibid. 36 (1979), 1-10.

[9] M. Malec and M. Rosati, Weak monotonicity for nonlinear systems of functionalfinite difference inequalities of parabolic type, Rend Mat. (7) 3 (1983), 157-170.

[10] M. Malec et A. Schiaffino, Méthode aux différences finies pour une équation nonlinéaire différentielle fonctionnelle du type parabolique avec une condition initiale de Cauchy, Boll. Un. Mat. Ital. B (7) 1 (1987), 99-109.

[11] C. V. Pao, Finite difference reaction-diffusion systems with coupled boundary conditions and time delays, J. Math. Anal. Appl. 272 (2002), 407-434; Corrigendum, ibid. 288 (2003), 870.

[12] —, Monotone methods for a finite difference system of reaction diffusion equations with time delay, Comput. Math. Appl. 36 (1998), 37-47.

[13] - Numerical methods for systems of nonlinear parabolic equations with time delays, J. Math. Anal. Appl. 240 (1999), 249-279.

[14] W. Voigt, Das Differenzenverfahren bei nichtlinearen parabolischen DifferentialFunktional-Gleichungen mit Rand-Funktional-Bedingungen, Beiträge Anal. 18 (1981), 91-98.

[15] —, Nonlinear parabolic differential-functional inequalities with boundary-functional conditions, ibid., 85-89.

[16] -, On finite-difference methods for parabolic functional-differential equations on unbounded domains, in: Numerical Methods and Applications (Sofia, 1989), Publ. House Bulgar. Acad. Sci., Sofia, 1989, 559-567.

Institute of Mathematics

University of Gdańsk

57, Wit Stwosz St.

80-952 Gdańsk, Poland

E-mail: karolina.kropielnicka@math.univ.gda.pl

Received 5.1.2007

and in final form 27.4.2007 